\title{
ORIGINAL ARTICLE \\ Contribución al conocimiento de la distribución y ecología de los murciélagos (Orden Chiroptera) en Castilla y León
}

\author{
Roberto J. Hermida Lorenzo ${ }^{1, *}$, Ledicia Santos Fernández ${ }^{1}$, Zeltia López Gallego ${ }^{1}$
}

\begin{abstract}
1 Morcegos de Galicia -
DROSERA, Magdalena G-2, 20

izq, 15320 As Pontes (A Coruña).
\end{abstract}

*Corresponding author e-mail: info@morcegosdegalicia.org

DOI: https://doi.org/10.14709/ BarbJ.11.1.2018.08

Keywords: murciélagos, Castilla y León, distribución, primeros datos, especies crípticas.

received: March, 7th 2018 accepted: August, 19th 2018

\begin{abstract}
RESUMEN
Entre los años 2011 y 2015 Drosera participó en varios proyectos con murciélagos en diferentes zonas de Castilla y León en los que se capturaron 1.071 ejemplares pertenecientes a 23 especies de murciélagos y se grabaron 15.274 archivos sonoros con vocalizaciones de murciélagos, en los que se pudo identificar de manera inequívoca 11 especies. En este manuscrito aportamos información para 24 especies. Se han obtenido las primeras citas confirmadas para algunas especies en el ámbito de estudio: Myotis cf. nattereri, Myotis escalerai y Eptesicus isabellinus. Para E. isabellinus se trata de la primera cita en Castilla y León y la localidad más septentrional en España. Para Myotis cf. nattereri y M. escalerai se aportan las primeras citas para algunas provincias. También se amplía notablemente el conocimiento de la distribución de algunas especies comunes, como Pipistrellus pygmaeus, y se ofrecen datos sobre la ecología de otras. En general se observa un patrón de distribución según el cual las especies más típicamente forestales aparecen únicamente en el perímetro montañoso de la comunidad autónoma, pero están ausentes de las zonas llanas desarboladas que ocupan buena parte de la zona central de Castilla y León y de los exiguos bosques de ribera que atraviesan estas zonas. Las zonas montañosas, en las que el relieve complejo determina mayor diversidad de hábitats y mantiene cierta extensión y naturalidad de diferentes tipos de bosque, se han mostrado más diversas en cuanto a especies de murciélagos.
\end{abstract}

\section{INTRODUCCIÓN}

En las últimas décadas se realizaron varios inventarios de quirópteros en distintos territorios de la península ibérica que representaron un notable avance en el conocimiento de la distribución y la ecología de los murciélagos ibéricos (Galán 1997, Flaquer et al. 2004, Lisón et al. 2011, Hermida et al. 2012, Molleda \& Fombellida 2018).

El conocimiento de la distribución y ecología de los murciélagos (Chiroptera) en Castilla y León experimentó un fuerte impulso con el proyecto Life/Naturaleza B43200/96/511, que entre 1998 y 2001 realizó un gran esfuerzo de campo para aportar información, entre otros aspectos, sobre la distribución de las especies de murciélagos en esta comunidad autónoma. Sin embargo, poco se ha avanzado desde entonces y apenas se ha recogido información sobre las especies que se han ido encontrando en la península ibérica en los últimos años a raíz de estudios sobre diversidad críptica (Ibáñez et al. 2006) como Myotis alcathoe, Myotis escalerai, Myotis cf. nattereri o Eptesicus isabellinus.

En el momento de la finalización del Atlas de distribución de las especies de Castilla y León (Fernández 2002) no se había descrito $M$. alcathoe todavía; los ejemplares de esta especie eran por entonces asignados a M. mystacinus, por lo que las citas de esta última especie podrían corresponder a cualquiera de las dos. En Palomo et al. (2007) no se incluyen citas de ejemplares analizados molecularmente para Castilla y León, por lo que, de nuevo, las citas podrían corresponder indistintamente a M. mystacinus o a $M$. alcathoe. Sobre la distribución de estas especies, apenas se han aportado unos pocos datos en los últimos años. Años más tarde, se confirmó molecularmente la presencia de $M$. alcathoe en los Ancares leoneses (Hermida et al. 2012, Hermida et al. 2013) y la de $M$. mystacinus en varios puntos de los sistemas montañosos que enmarcan la comunidad (Nogueras et al. 2013).

Tampoco se había puesto de manifiesto la diversidad dentro del complejo Myotis cf nattereri, ni en Fernández (2002) ni en Palomo et al. (2007). En la actualidad se sabe que Myotis nattereri 'sensu stricto' no está presente en la península ibérica y los ejemplares asignados anteriormente a esta especie pertenecen, en realidad, bien a Myotis escalerai, bien a una segunda especie identificada mediante caracteres moleculares, pero pendiente todavía de descripción formal (Salicini et al. 2011, 2013). Únicamente se había confirmado molecularmente la presencia de $M$. escalerai en Castilla y León en los Ancares leoneses (Hermida 
et al. 2012) y de M. escalerai y M. cf nattereri en los jardines del palacio de La Granja, Segovia (de Paz et al. 2016) y en los Montes de Valsaín (de Paz et al. 2017), estos últimos fuera del ámbito de este trabajo.

De igual modo, Fernández (2002) incluyó Pipistrellus pygmaeus únicamente en una cuadrícula en el sureste de Castilla y León, pero en el resto de la comunidad autónoma asignó todos los ejemplares a $P$. pipistrellus. Tampoco en Palomo et al. (2007) se diferencia entre ambas especies, por lo que las citas incluidas en estas publicaciones podrían corresponder a cualquiera de ellas. Más recientemente, se reportó la presencia de $P$. pygmaeus en la montaña segoviana (de Paz et al. 2016, de Paz et al. 2017).

A fin de contribuir al mejor conocimiento de la distribución y la ecología de los murciélagos en Castilla y León, en este trabajo aportamos información sobre distribución de especies obtenida a lo largo de varios muestreos enmarcados en diferentes proyectos realizados en los años 2011, 2014 y 2015 en esta comunidad.

\section{METODOLOGÍA}

Los muestreos realizados en Castilla y León se corresponden con tres proyectos diferentes desarrollados en los años 2011, 2014 y 2015.

Entre el 10 de mayo y el 30 de septiembre del año 2011, como parte del proyecto "Murciélagos en soutos: un ejemplo de biodiversidad asociada a bosques culturales" se muestrearon con redes de niebla ocho localidades en la sierra de Ancares (León), pertenecientes a tres cuadrículas UTM 10x10. El objetivo de estos muestreos era la captura y estudio de especies forestales, por lo que el esfuerzo y métodos se dirigieron a ese fin.

Los muestreos realizados durante el año 2014 tenían por objeto caracterizar el uso por murciélagos de los hábitats de ribera presentes en los LICs de ambientes mediterráneos de Castilla y León, como parte del Proyecto LIFE11 NAT ES/699 MEDWETRIVERS, por lo que sus resultados están sesgados hacia las especies más frecuentes en este tipo de ambientes. Los muestreos se realizaron entre el 1 de junio y el 31 de agosto. El ámbito del proyecto incluía 234 cuadrículas UTM de 10×10 km, de las que se seleccionaron 53. En cada una de estas 53 cuadrículas se realizaron tres estaciones de muestreo, lo que conlleva un total de 159 puntos de muestreo. En 149 estaciones se muestreó conjuntamente con redes de niebla y detectores de ultrasonidos, y en 10 únicamente con detectores de ultrasonidos (Fig. 1).

La distribución de los tres puntos de muestreo en cada cuadrícula se realizó intentando abarcar la diversidad de hábitats y la potencial diversidad de especies presentes en el LIC. En conjunto, se intentó obtener, en cada cuadrícula, una representación de la diversidad de especies de murciélagos presentes en los hábitats que conforman el LIC.

Por último, en septiembre de 2015 se muestrearon con redes de niebla siete localidades en cinco cuadrículas UTM 10x10 de la provincia de León con el objetivo de capturar diferentes especies del género Myotis, como parte del

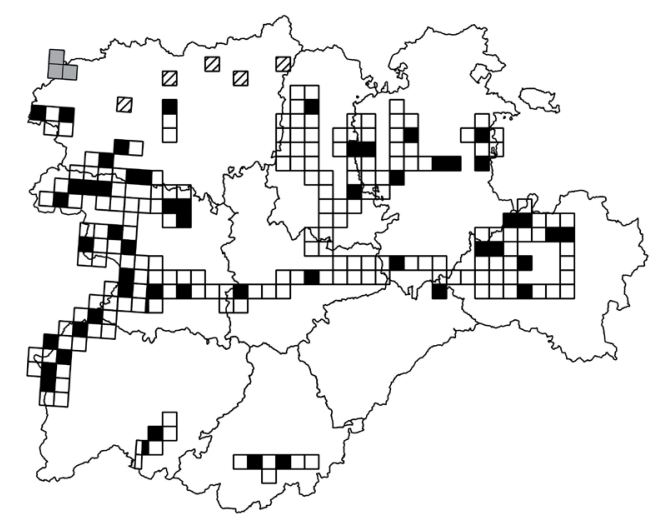

Cuadrículas UTM10x10 muestreadas en 2011

Cuadrículas UTM10x10 muestreadas en el Life MedWetRivers (2014)

Resto del ámbito de estudio del Life MedWetRivers (2014)

//. Cuadrículas UTM10x10 muestreadas en 2015

Fig. 1 - Ámbito de trabajo y cuadrículas muestreadas en cada año y proyecto.

trabajo de campo del proyecto Global Change Genetics (www.globalchangegenetics.org).

\section{Estaciones de captura}

Para las capturas se utilizaron redes de niebla de nylon y poliéster de entre 2 y 18 metros de longitud (Ecotone, Polonia). Los murciélagos capturados fueron guardados momentáneamente en bolsas de algodón, en espera de ser procesados. De cada ejemplar se anotó la especie, el sexo, la edad (se diferenciaron únicamente los ejemplares del año de otras edades), la medida del antebrazo, el peso, el estado reproductor y el desgaste dentario, además de las observaciones pertinentes en cada caso. Para la anotación del estado reproductor y el desgaste dentario se utilizaron las categorías propuestas por Haarsma (2008).

En el caso de especies pertenecientes a grupos con diversidad críptica, se tomó una muestra de patagio que se conservó en etanol $70 \%$ para posterior confirmación molecular de la especie. En concreto, se tomaron biopsias de los complejos específicos: Myotis mystacinus/alcathoe, Myotis escalerai/cf. nattereri, Eptesicus serotinus/isabellinus, Plecotus auritus/austriacus; y de algunos ejemplares de los complejos $P$. pipistrellus/pygmaeus y Myotis myotis/blythii en los que existían dudas sobre su identificación.

Los ejemplares fueron liberados en el mismo punto de captura, una vez procesados. El análisis de ADN se realizó en el Laboratorio de Ecología Molecular (LEM) de la Estación Biológica de Doñana. En el laboratorio se extrajo el ADN de las biopsias de ala siguiendo Higuchi et al. (1988). Dependiendo de las especies se amplificaron bien un fragmento de la subunidad 1 del gen mitocondrial $\mathrm{NADH}$ deshidrogenasa (ND1) o bien un fragmento del gen del Citocromo b (ctyb), utilizando los cebadores ND1-F2 y ND1-R (Kawai et al. 2002) o Molcit-F (Ibáñez et al. 2006) y MVZ-16 (Smith \& Patton 1993). Las condiciones de amplificación y secuenciación siguieron las descritas en Ibáñez et al. (2006). Los fragmentos se secuenciaron directamente a partir de producto de PCR purificado usando un secuenciador automático ABI 3100 (Applied Biosystems, Warrington, RU) y siguiendo los protocolos del fabricante. Las secuencias parciales se alinearon y editaron con el 
programa Geneious R7 (Biomatters Ltd.) y se inspeccionaron visualmente. Para fines de identificación, las secuencias obtenidas se compararon con secuencias disponibles en la base de datos GenBank (http://www.ncbi.nlm.nih.gov/ GenBank) utilizando la herramienta BLAST o bien mediante comparación directa con la colección de secuencias almacenadas por el grupo de estudio de murciélagos de la Estación Biológica de Doñana (Sevilla).

\section{Estaciones de muestreo acústico}

Para las estaciones de muestreo acústico se utilizaron detectores Pettersson D240x (Pettersson Elektronik, Suecia) y grabadoras digitales Zoom H2 y Zoom H2n (Zoom Corporation, Japón). Los detectores se configuraron para realizar grabaciones automáticas en tiempo expandido (factor $\times 10$ ) de 1,7 segundos de duración. La sensibilidad del disparador se ajustó al máximo, de modo que se grabase de manera continua un archivo de 1,7 segundos cada 17 segundos, tiempo empleado para transferir el archivo en tiempo expandido a la grabadora. La grabadora se configuró en modo Autorec para que crease un archivo de sonido cada vez que el detector generaba una grabación. En cada punto de muestreo, el detector se situó aproximadamente a 1,5 metros de altura, sujeto a un poste o árbol que no entorpeciese la recepción con su follaje.

Los archivos grabados fueron procesados con Sonobat (Sonobat, USA). En primer lugar se utilizó Sonobat Batch Attributer 6.4 para renombrar los archivos y descartar aquellos que no contenían pulsos de murciélagos (se aplicó el filtro "high grade" para seleccionar únicamente archivos con llamadas nítidas y fuertes). A continuación, se utilizó la función automática Sonobatch de la utilidad Sonobat 3.1.8p para analizar los pulsos de murciélagos. Se analizaron un máximo de ocho pulsos por archivo y para cada uno se obtuvieron los datos de frecuencia de máxima energía, duración, pendiente, ancho de banda e intervalos, descartando los pulsos con una calidad inferior a 0,70. Con estos datos se realizó una primera asignación automática de las llamadas a diferentes grupos de especies, asignación que fue posteriormente revisada de manera manual con Sonobat 3.1.8p para su confirmación o descarte. En la identificación específica se utilizaron tanto los sonidos de la fonoteca propia de Drosera (no publicado), como los criterios recogidos en Barataud (2012) y se descartó cualquier archivo que presentase dudas en su asignación específica.

\section{Refugios}

Aunque no se realizó una búsqueda sistemática de refugios, se revisaron aquellas construcciones próximas a los puntos de muestreo que mostraron potencial para acoger murciélagos.

\section{RESULTADOS Y DISCUSIÓN}

Este estudio aporta información para un total de 24 especies, de las cuales 23 han sido capturadas y, en algunos casos, también detectadas acústicamente, y una (Tadarida teniotis) ha sido detectada únicamente por sus emisiones acústicas.
Entre los años 2011 y 2015 se capturaron 1.071 ejemplares pertenecientes a 23 especies.

En las estaciones de muestreo acústico se grabaron 15.274 archivos sonoros con vocalizaciones de murciélagos. Se pudieron identificar de manera inequívoca 11 especies; los registros identificados a nivel de género o como pares de especies no se han reflejado en este artículo.

Se han obtenido las primeras citas en el ámbito de estudio, confirmadas mediante análisis molecular, para las especies: $M$. cf. nattereri, $M$. escalerai y $E$. isabellinus. Para $E$. isabellinus se trata de la primera cita en Castilla y León. Para Myotis cf. nattereri y M. escalerai se aportan las primeras citas para algunas provincias. $P$. pygmaeus había sido citada únicamente en una localidad en la comunidad (Fernández 2002), pero se revela como una especie común en buena parte del territorio.

A continuación ofrecemos información más detallada para cada especie y los mapas de distribución de las citas aportadas.

\section{Rhinolophus ferrumequinum (Schreber, 1774)}

Se ha detectado en diez cuadrículas, seis mediante capturas y cuatro en registros acústicos (Fig. 2). Se aportan tres nuevas cuadrículas dentro del área de distribución previamente conocida. Se ha identificado y censado un refugio de cría en la provincia de Soria, en las inmediaciones de Berlanga de Duero el día 1/07/2014. En dicha fecha se confirmó la presencia de algunos jóvenes del año de edad avanzada, junto a una mayoría de crías no volanderas que permanecían agrupadas tras la emergencia de los adultos.

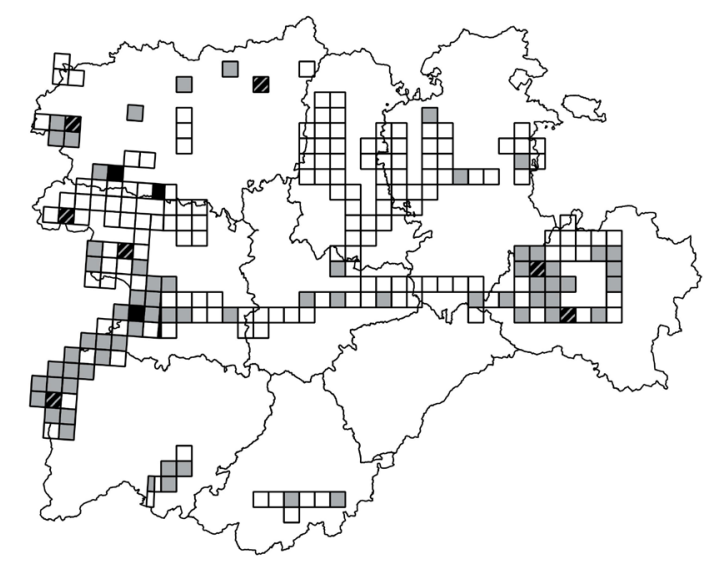

$$
\begin{aligned}
& \text { Cuadrículas con presencia de la especie según bibliografía } \\
& \text { Nuevas cuadrículas aportadas en el presente trabajo } \\
& \text { Cuadrículas en las que se había citado la especie en bibliografía } \\
& \text { y se ha vuelto a detectar durante el presente estudio }
\end{aligned}
$$

Fig. 2 - Distribución de Rhinolophus ferrumequinum en el área de estudio.

\section{Rhinolophus hipposideros (Bechstein, 1800)}

Detectado en 11 cuadrículas (Fig. 3): una mediante capturas, cinco en registros acústicos y otras cinco en refugio. Seis de estas cuadrículas son nuevas, aunque se encuentran dentro del área de distribución conocida. 


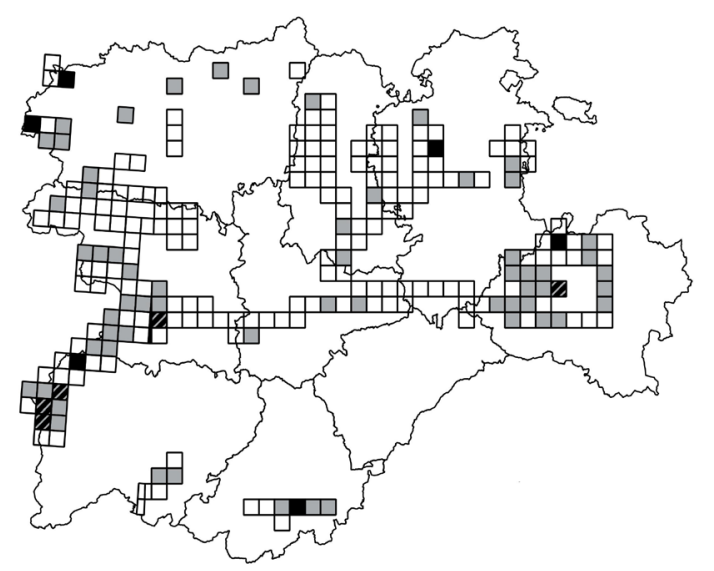

- Cuadrículas con presencia de la especie según bibliografía

- Nuevas cuadrículas aportadas en el presente trabajo

Cuadrículas en las que se había citado la especie en bibliografía y se ha vuelto a detectar durante el presente estudio

Fig. 3 - Distribución de Rhinolophus hipposideros en el área de estudio.

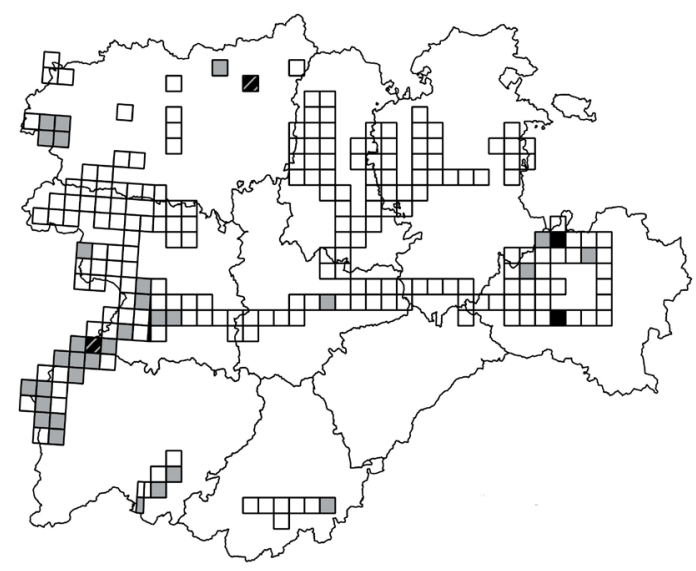

- Cuadrículas con presencia de la especie según bibliografía

- Nuevas cuadrículas aportadas en el presente trabajo

Cuadrículas en las que se había citado la especie en bibliografía

y se ha vuelto a detectar durante el presente estudio

Fig. 4 - Distribución de Rhinolophus euryale en el área de estudio.

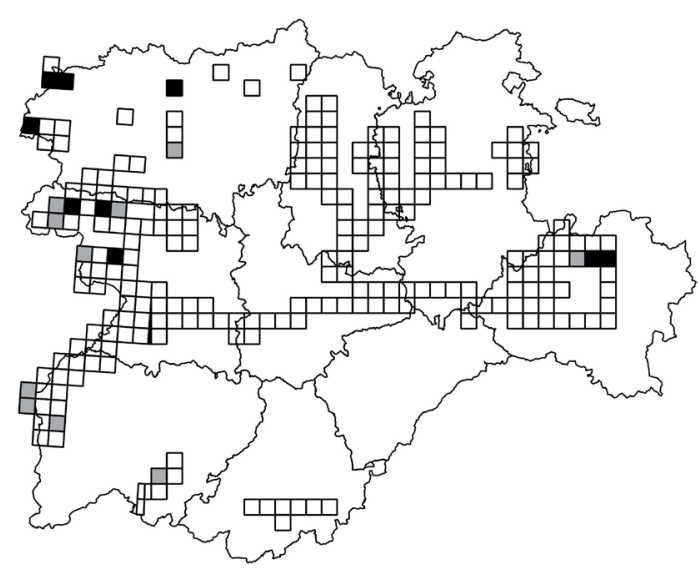

- Cuadrículas con presencia de la especie según bibliografía

- Nuevas cuadrículas aportadas en el presente trabajo

Cuadrículas en las que se había citado la especie en bibliografía

y se ha vuelto a detectar durante el presente estudio

Fig. 5 - Distribución de Myotis mystacinus en el área de estudio.

\section{Rhinolophus euryale Blasius, 1853}

Detectado mediante captura en una cuadrícula y por sondeos acústicos en otras tres (Fig. 4). Dos de estas cuadrículas son nuevas, aunque dentro del área de distribución conocida.

\section{Myotis mystacinus (Kuhl, 1817)}

Capturado en nueve cuadrículas, todas nuevas, dentro del área conocida de distribución (Fig. 5). No se ha podido confirmar su presencia en los enclaves más meridionales citados en la bibliografía, como el LIC Arribes del Duero o el LIC Riberas del río Alagón y afluentes (Hernández 2010). Parece una especie común en la montaña zamoranoleonesa y en el norte de la provincia de Soria. En el curso alto del Duero, en la provincia de Soria, se han capturado hembras gestantes en una zona de mosaico silvopastoral y bosque aluvial cercana a la localidad de Hinojosa de la Sierra, a principios de julio de 2014. En la península ibérica se asocia esta especie con hábitats forestales de montaña (Nogueras et al. 2013), aunque también se ha detectado en bosques aluviales y paisajes agrícolas a baja altitud en Galicia (Drosera 2014); fuera de la península ibérica se ha detectado en hábitats muy abiertos como estepas y semidesiertos (Hutson et al. 2008); utiliza frecuentemente construcciones humanas para refugiarse.

\section{Myotis alcathoe Helversen y Heller, 2001}

Se ha detectado esta especie en varias localidades de la provincia de León correspondientes a dos cuadrículas: valle de Burbia y valle de Sortes (LIC Sierra de Ancares) y Boca de Huérgano (Fig. 6). No obstante, la información correspondiente a los muestreos del año 2011 en la sierra de Ancares ya fue expuesta en Hermida et al. (2012), por lo que se recoge en el mapa como información bibliográfica y se aporta una única cuadrícula nueva, correspondiente a los muestreos realizados en 2015 .

M. alcathoe es una especie de reciente descripción, de la que apenas estamos comenzando a conocer su ecología. En su rango de distribución conocido en Europa aparece principalmente en bosques con un elevado grado de madurez (Niermann et al. 2007, Coronado et al. 2017). Aunque esta especie no ha sido detectada hasta el momento en la montaña soriana o burgalesa, se considera probable su presencia allí, pues el hábitat es adecuado para la especie y existen poblaciones muy cercanas en La Rioja (AguirreMendi et al. 2004). También ha sido citada en hábitats similares en Galicia (Hermida et al. 2012).

\section{Myotis escalerai Cabrera, 1904}

Capturado en cinco cuadrículas, que representan las primeras citas confirmadas para esta especie en el ámbito de estudio (Fig. 7). Probablemente es una especie más común en el territorio estudiado de lo que permiten constatar los resultados. $M$. escalerai se refugia tanto en cavidades (Quetglas 2016) como en construcciones (Hermida \& Arzúa 2012). Se han encontrado en varias ocasiones colonias de cría en construcciones sin terminar. En estas situaciones, la colonia es especialmente vulnerable pues se puede esperar la reactivación de las obras o su derrumbe en cualquier momento. 


\section{Myotis cf. nattereri (Kuhl, 1817)}

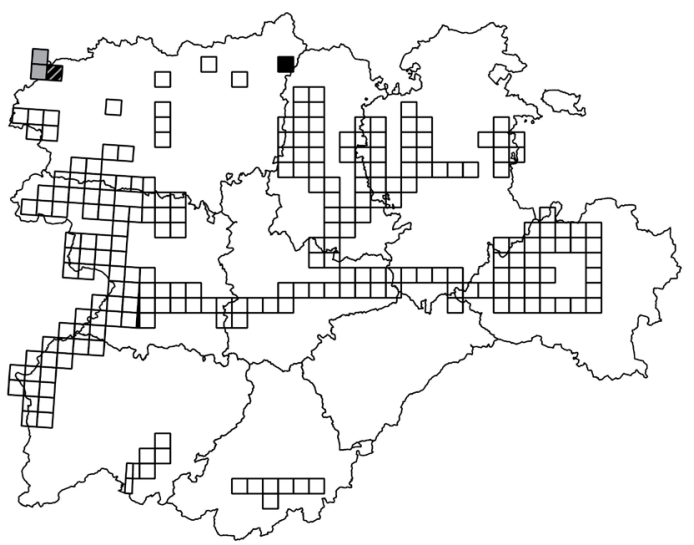

Cuadrículas con presencia de la especie según bibliografía

- Nuevas cuadrículas aportadas en el presente trabajo

Cuadrículas en las que se había citado la especie en bibliografía

y se ha vuelto a detectar durante el presente estudio

Fig. 6 - Distribución de Myotis alcathoe en el área de estudio.

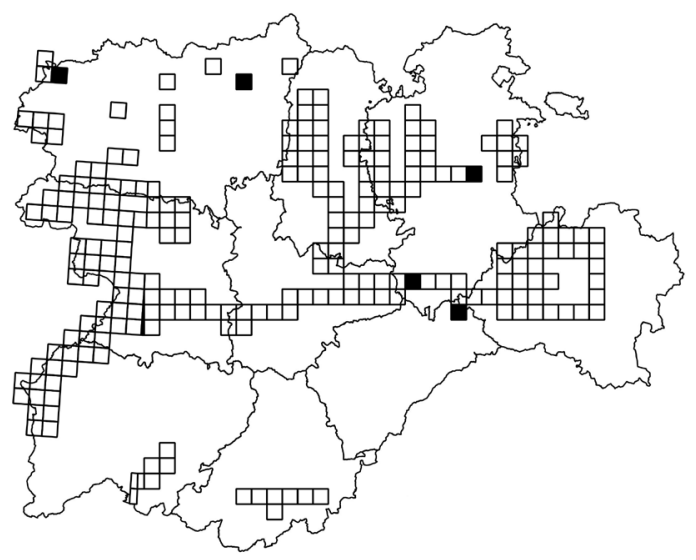

- Cuadrículas con presencia de la especie según bibliografía

- Nuevas cuadrículas aportadas en el presente trabajo

Cuadrículas en las que se había citado la especie en bibliografía

y se ha vuelto a detectar durante el presente estudio

Fig. 7 - Distribución de Myotis escalerai en el área de estudio.

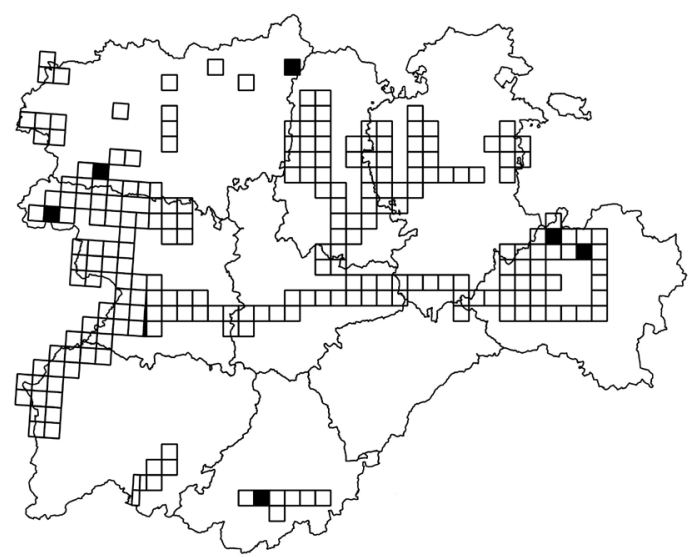

- Cuadrículas con presencia de la especie según bibliografía

- Nuevas cuadrículas aportadas en el presente trabajo Cuadrículas en las que se había citado la especie en bibliografía y se ha vuelto a detectar durante el presente estudio

Fig. 8 - Distribución de Myotis cf. nattereri en el área de estudio.
Capturado en seis cuadrículas que representan las primeras citas confirmadas de la especie en el ámbito de estudio y de las pocas que existen para Castilla y León (Fig. 8). Especie presente en las zonas de montaña de la periferia de la comunidad. En el curso alto del Duero, en la provincia de Soria, se capturaron hembras gestantes en una zona de mosaico silvopastoral y bosque aluvial cercana a la localidad de Hinojosa de la Sierra, a principios de julio de 2014. Apenas se comienza a disponer de información sobre la distribución y la ecología de Myotis cf. nattereri, cuya descripción y nombre definitivo están todavía pendiente de publicación. Parece ser una especie ligada a los sistemas montañosos del norte y centro peninsular, de hábitos eminentemente forestales (Salicini et al. 2013).

\section{Myotis myotis (Borkhausen, 1797)}

Ha sido capturado en 12 cuadrículas, ocho de ellas nuevas (Fig. 9). Especie común en la mayor parte del ámbito de estudio. El hecho de que se refugie habitualmente entre las piedras de viejos puentes ha facilitado su captura en estaciones situadas bajo estas estructuras.

\section{Myotis emarginatus (Geoffroy, 1806)}

Se ha capturado esta especie en cuatro cuadrículas, todas ellas nuevas, si bien en dos LICs en los que la presencia de la especie era ya conocida (Fig. 10). Se ha identificado un refugio de cría compartido con Rhinolophus ferrumequinum cercano a la localidad de Berlanga de Duero en el que se han contado un total de 65 ejemplares adultos de $M$. emarginatus. En Extremadura, Goiti et al. (2011) pusieron de manifiesto la importancia de los bosques de ribera para $M$. emarginatus, aunque también utiliza pinares y otros hábitats complejos para alimentarse, principalmente de arañas. En Cataluña, selecciona preferentemente bosques de coníferas y zonas mixtas con olivares tradicionales (Flaquer et al. 2008), mientras que en el sur de Alemania parece estar ligado a zonas ganaderas, entrando con frecuencia en establos para cazar (Steck et al. 2006).

\section{Myotis bechsteinii (Kuhl, 1817)}

Capturado en dos cuadrículas, en el Sistema Central, en sendos LICs en los que no había sido citado con anterioridad: Riberas del río Alagón y afluentes (Salamanca) y Riberas del río Alberche y afluentes (Ávila) (Fig. 11). En general, esta especie se mueve en el entorno inmediato de sus refugios (Fiztsimons et al. 2002, Salsamendi et al. 2007, López \& Hermida 2016), por lo que en su conservación es fundamental la adecuada gestión del hábitat en un radio de $1 \mathrm{~km}$ de los refugios. Aún sin conocer la exacta ubicación de las zonas-refugio, se pueden fomentar de modo especial estas medidas en el entorno de las zonas en las que se han detectado hembras en época reproductora.

\section{Myotis daubentonii (Kuhl, 1817)}

Ha sido capturado en 42 cuadrículas, 19 de las cuales son nuevas (Fig. 12). Especie abundante en todo el ámbito de estudio, siempre que existan cursos de agua permanentes. Aunque en esta especie se produce una segregación sexual 


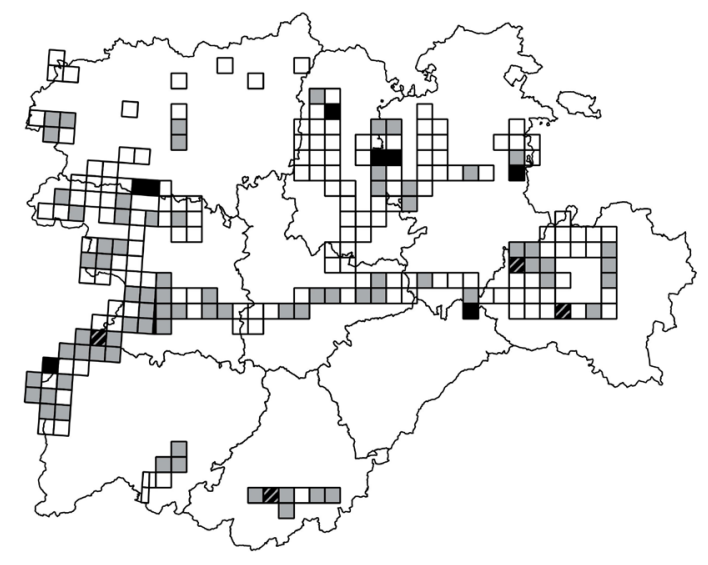

- Cuadrículas con presencia de la especie según bibliografía

- Nuevas cuadrículas aportadas en el presente trabajo

Cuadrículas en las que se había citado la especie en bibliografía

y se ha vuelto a detectar durante el presente estudio

Fig. 9 - Distribución de Myotis myotis en el área de estudio.

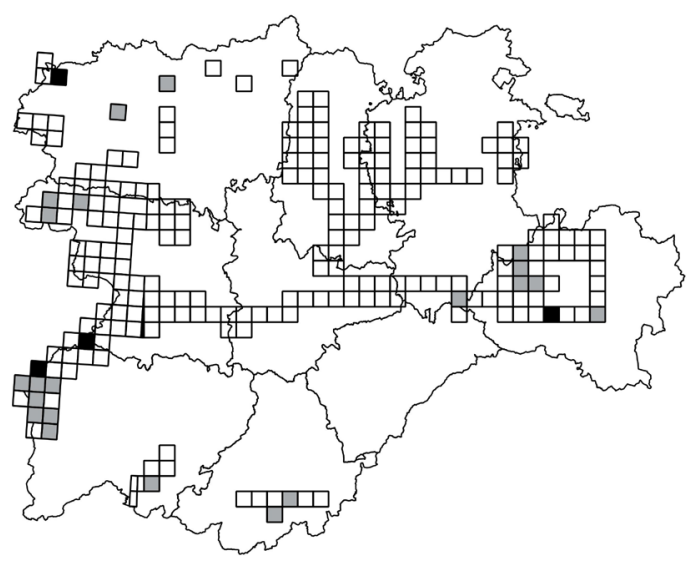

Cuadrículas con presencia de la especie según bibliografía

- Nuevas cuadrículas aportadas en el presente trabajo

Cuadrículas en las que se había citado la especie en bibliografía

y se ha vuelto a detectar durante el presente estudio

Fig. 10 - Distribución de Myotis emarginatus en el área de estudio.

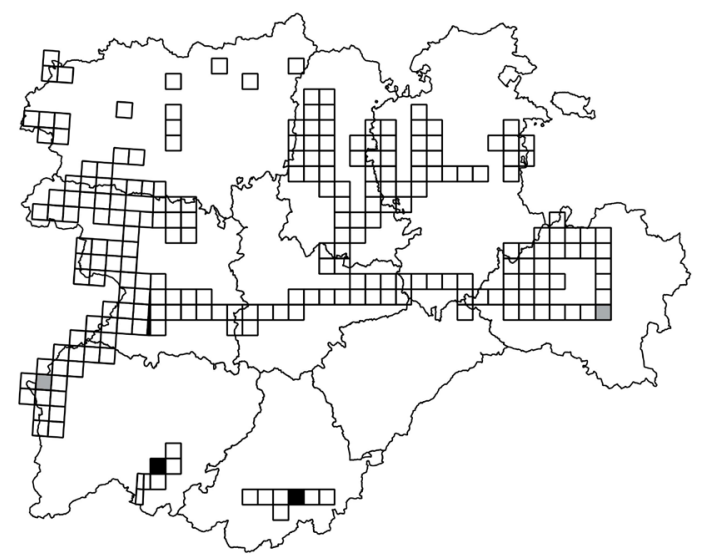

- Cuadrículas con presencia de la especie según bibliografía

- Nuevas cuadrículas aportadas en el presente trabajo

Cuadrículas en las que se había citado la especie en bibliografía

y se ha vuelto a detectar durante el presente estudio

Fig. 11 - Distribución de Myotis bechsteinii en el área de estudio.

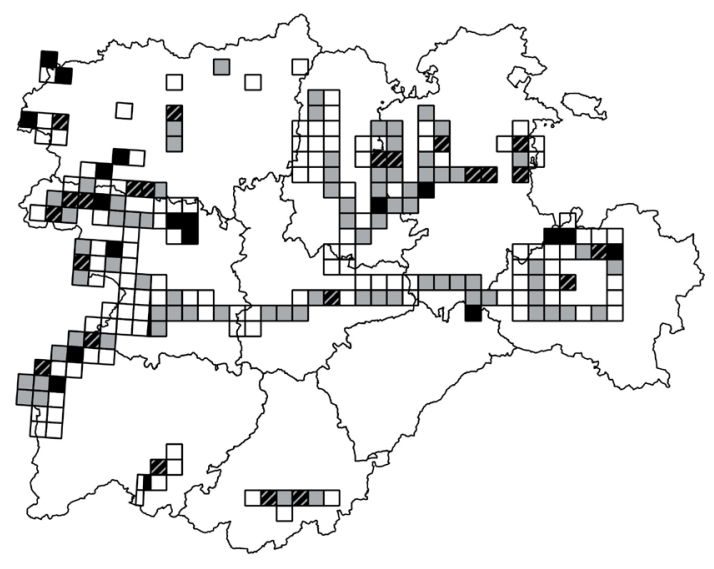

- Cuadrículas con presencia de la especie según bibliografía

- Nuevas cuadrículas aportadas en el presente trabajo

Cuadrículas en las que se había citado la especie en bibliografía

y se ha vuelto a detectar durante el presente estudio

Fig. 12 - Distribución de Myotis daubentonii en el área de estudio.

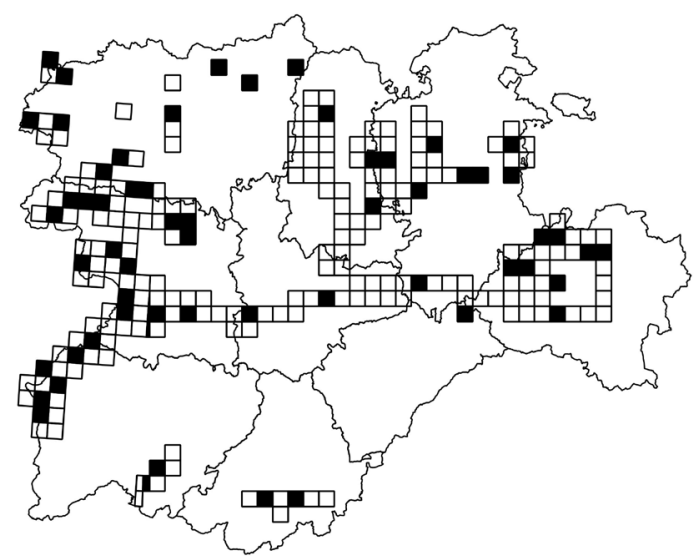

- Cuadrículas con presencia de la especie según bibliografía

- Nuevas cuadrículas aportadas en el presente trabajo

Cuadrículas en las que se había citado la especie en bibliografía

y se ha vuelto a detectar durante el presente estudio

Fig. 13 - Distribución de Pipistrellus pipistrellus en el área de estudio.

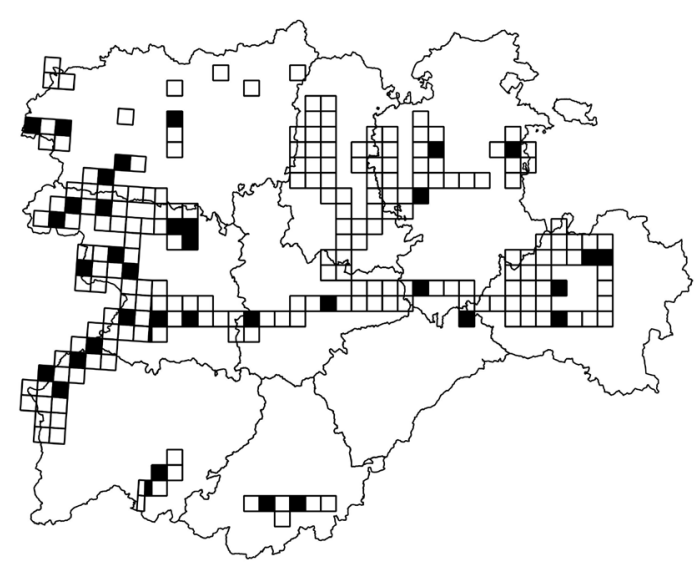

- Cuadrículas con presencia de la especie según bibliografía

- Nuevas cuadrículas aportadas en el presente trabajo

Cuadrículas en las que se había citado la especie en bibliografía

y se ha vuelto a detectar durante el presente estudio

Fig. 14 - Distribución de Pipistrellus pygmaeus en el área de estudio. 
durante la época reproductora y las hembras tienden a ocupar partes más bajas y térmicas de los valles fluviales (Altringham et al 1997, Leuzinger \& Brossard 1994, Russo 2002), se capturaron hembras reproductoras (lactantes y postlactantes) y juveniles en el río Alberche entre los 1.250 y los $1.430 \mathrm{msnm}$, con temperaturas mínimas que oscilaron entre los 2,5 - 0ㄷ, a mediados de agosto de 2014 .

M. daubentonii presenta dos morfotipos en la península ibérica, cuya implicación taxonómica no está clara: M.d. daubentonii y M.d. nathalinae (Tupinier 1977, Juste 2017). Ambos morfotipos se diferencian externamente en la coloración y el tamaño, siendo M.d. nathalinae más oscuro, menos contrastado y de menor tamaño que M.d. daubentonii. Ambos morfotipos se encuentran presentes en simpatría en la mayor parte de las localidades. La presencia de M.d. nathalinae en la Península Ibérica se ha relacionado con la existencia de un "refugio dentro de refugio" durante los últimos máximos glaciares (Simões et al. 2007). Independientemente de la interpretación taxonómica que se realice de la variabilidad observada, ésta representa un patrimonio genético que merece ser conservado, además de un interesante campo de investigación evolutiva.

\section{Pipistrellus pipistrellus (Schreber, 1774)}

Se ha capturado en 46 cuadrículas y detectado acústicamente en 12 más (Fig. 13). Ampliamente extendido y abundante en toda el área de estudio. La información bibliográfica previa no distingue claramente entre $P$. pipistrellus y $P$. pygmaeus. Por tanto, para evitar confusiones, se ha optado por representar únicamente las citas confirmadas en este estudio. Se identificó una pequeña casa en el municipio de Cordovilla la Real (Palencia) del que emergieron 147 ejemplares a principios de agosto de 2014, entre adultos y algunos jóvenes del año.

\section{Pipistrellus pygmaeus (Leach, 1825)}

Capturado en 14 cuadrículas y detectado acústicamente en otras 22 (Fig. 14). Ampliamente extendido y abundante en los bosques aluviales de la meseta. Parece más escasa e incluso ausente en algunas cuadrículas prospectadas en las provincias de Burgos y Palencia. La información bibliográfica existente para el ámbito de estudio no distingue claramente entre $P$. pipistrellus y $P$. pygmaeus. Por tanto, para evitar confusiones, se ha optado por representar únicamente las citas confirmadas en este estudio. En el LIC Riberas de Castronuño se localizó un refugio, formado por una caseta que alberga un transformador eléctrico y una gran grieta en un árbol vecino, del que emergieron a mediados de agosto de 2014 más de 1.300 ejemplares, entre adultos y jóvenes de ese mismo año.

\section{Pipistrellus kuhlii (Kuhl, 1817)}

Se ha capturado en cinco cuadrículas y detectado acústicamente en otras 23 (Fig. 15). De todas ellas, 20 son nuevas. Se cita por primera vez en los LIC Cañón del río Lobos y Riberas del río Sil y afluentes. Es común en todo el LIC Arribes del Duero y en la parte soriana del LIC Riberas del río Duero y afluentes, en los que había sido escasamente citada. Especialmente abundante en el valle del Duero.

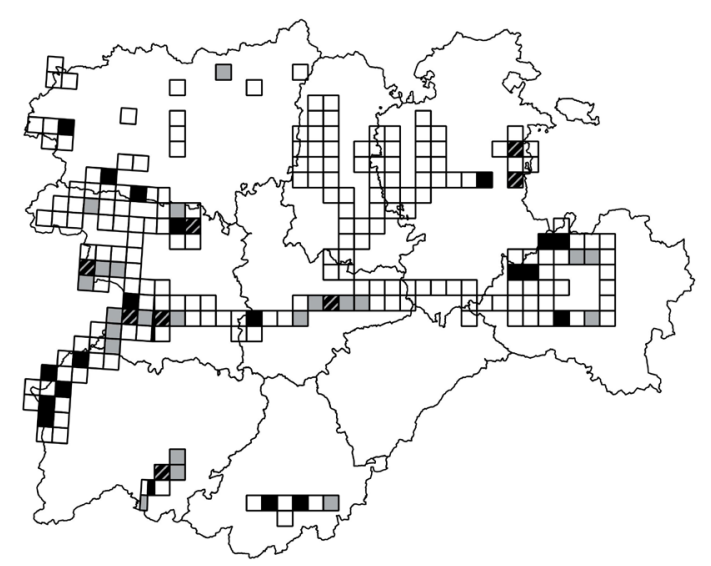

Cuadrículas con presencia de la especie según bibliografía

- Nuevas cuadrículas aportadas en el presente trabajo Cuadrículas en las que se había citado la especie en bibliografía y se ha vuelto a detectar durante el presente estudio

Fig. 15 - Distribución de Pipistrellus kuhlii en el área de estudio.

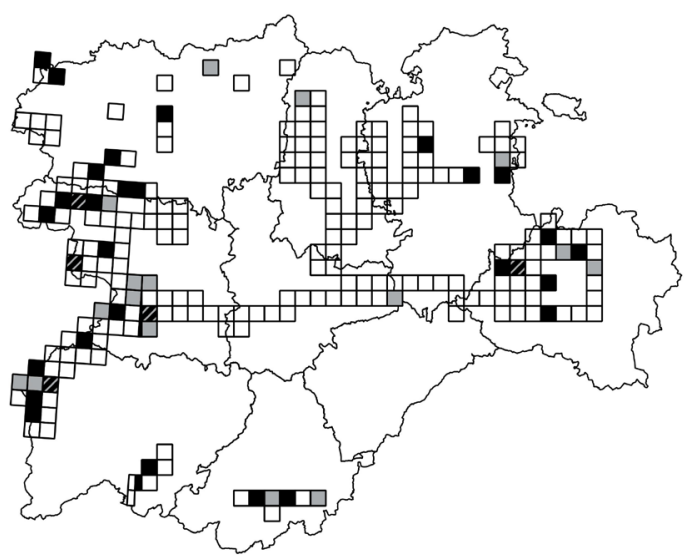

- Cuadrículas con presencia de la especie según bibliografía

- Nuevas cuadrículas aportadas en el presente trabajo Cuadrículas en las que se había citado la especie en bibliografía y se ha vuelto a detectar durante el presente estudio

Fig. 16 - Distribución de Hypsugo savii en el área de estudio.

\section{Hypsugo savii Bonaparte, 1837}

Capturas en 17 cuadrículas y registros sonoros en 16; 28 de esas cuadrículas son nuevas (Fig. 16). Es común, e incluso abundante, en las zonas orográficamente más complejas, mientras que está ausente en las zonas llanas.

\section{Eptesicus serotinus Schreber, 1774}

Se capturó en 11 cuadrículas, ocho de ellas nuevas. Se obtuvieron registros sonoros en 26 cuadrículas, 16 de ellas nuevas (Fig. 17). Se observó la emergencia de un gran número de ejemplares de esta especie en el pueblo de Monleón (Salamanca) aunque no se pudo identificar el refugio del que procedían. Dada la constatación de la presencia de $E$. isabellinus en el sur de la comunidad, como se comentará a continuación, algunos registros sonoros en las cuadrículas más meridionales podrían corresponder a $E$. isabellinus. 


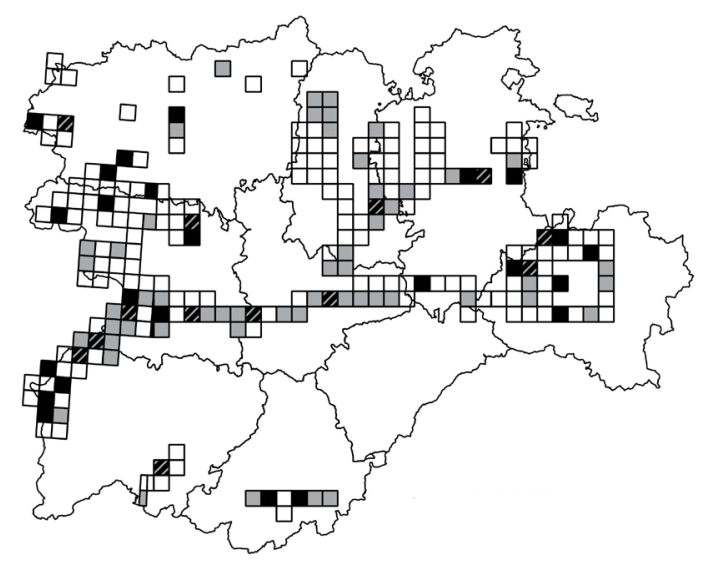

- Cuadrículas con presencia de la especie según bibliografía

- Nuevas cuadrículas aportadas en el presente trabajo

Cuadrículas en las que se había citado la especie en bibliografía

y se ha vuelto a detectar durante el presente estudio

Fig. 17 - Distribución de Eptesicus serotinus en el área de estudio.

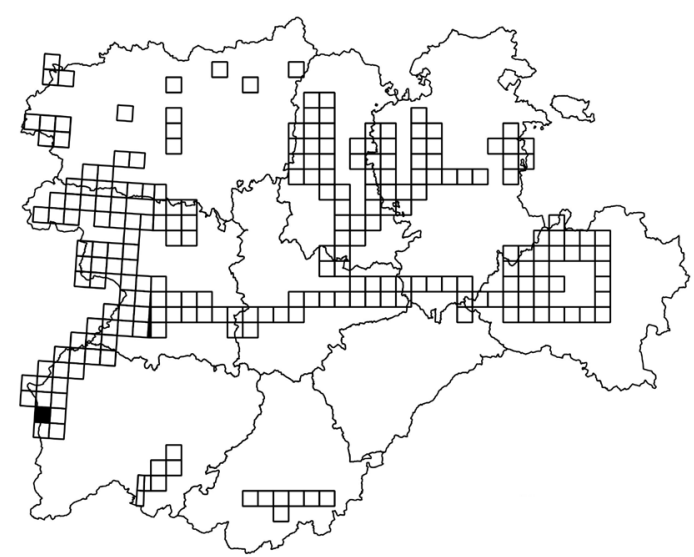

Cuadrículas con presencia de la especie según bibliografía

- Nuevas cuadrículas aportadas en el presente trabajo

Cuadrículas en las que se había citado la especie en bibliografía

y se ha vuelto a detectar durante el presente estudio

Fig. 18 - Distribución de Eptesicus isabellinus en el área de estudio.

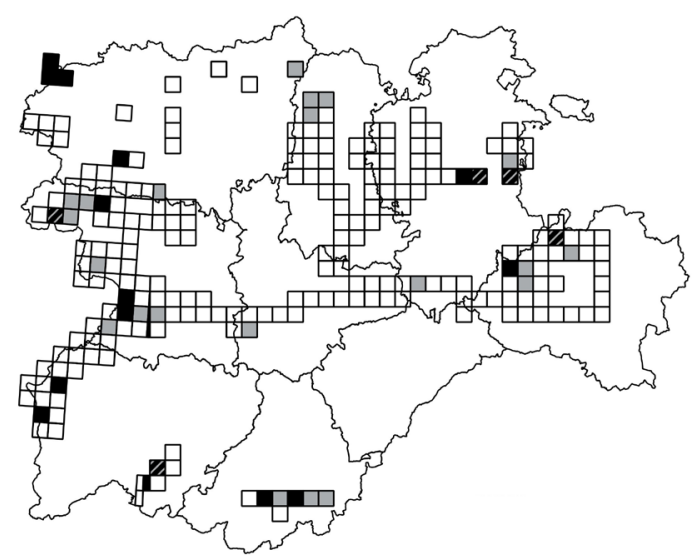

- Cuadrículas con presencia de la especie según bibliografía

- Nuevas cuadrículas aportadas en el presente trabajo

Cuadrículas en las que se había citado la especie en bibliografía

y se ha vuelto a detectar durante el presente estudio

Fig. 19 - Distribución de Nyctalus leisleri en el área de estudio.

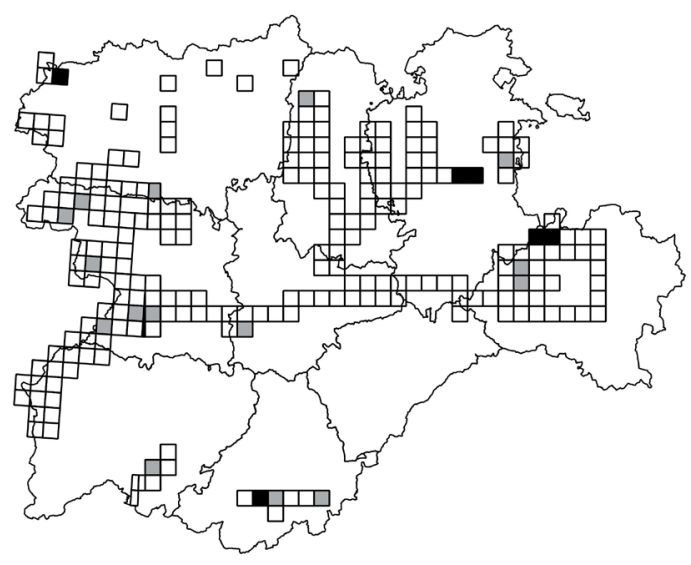

- Cuadrículas con presencia de la especie según bibliografía

- Nuevas cuadrículas aportadas en el presente trabajo

Cuadrículas en las que se había citado la especie en bibliografía

y se ha vuelto a detectar durante el presente estudio

Fig. 20 - Distribución de Nyctalus lasiopterus en el área de estudio.

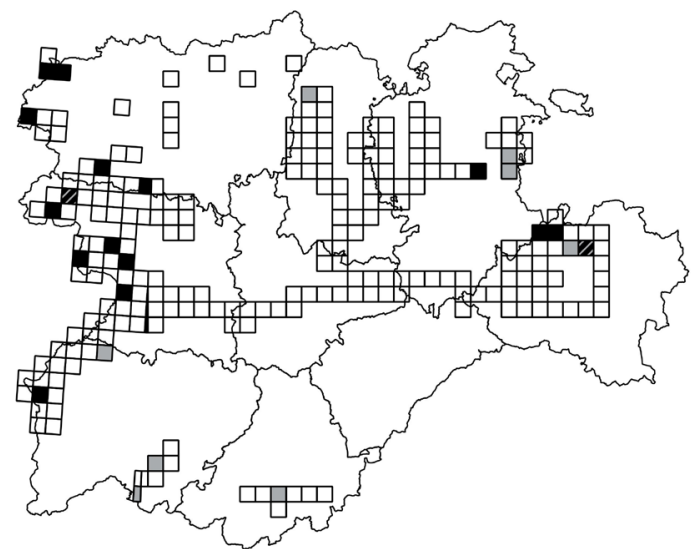

- Cuadrículas con presencia de la especie según bibliografía

- Nuevas cuadrículas aportadas en el presente trabajo

Cuadrículas en las que se había citado la especie en bibliografía

$v$ se ha vuelto a detectar durante el presente estudio

Fig. 21 - Distribución de Barbastella barbastellus en el área de estudio.

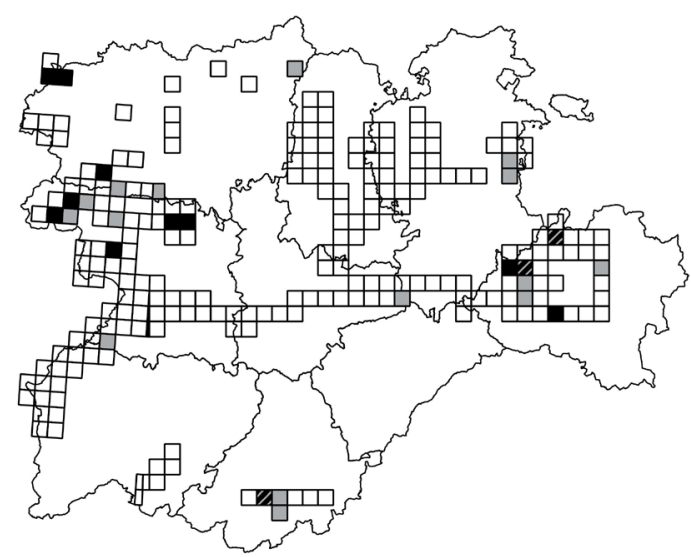

- Cuadrículas con presencia de la especie según bibliografía

- Nuevas cuadrículas aportadas en el presente trabajo

Cuadrículas en las que se había citado la especie en bibliografía y se ha vuelto a detectar durante el presente estudio 


\section{Eptesicus isabellinus Temminck, 1840}

Una única captura, en el valle del río Águeda, municipio de Puerto Seguro (Salamanca) (Fig. 18). Supone la primera cita para Castilla y León y la localidad más septentrional en España, aunque en Portugal ya se localizado más al norte (Barros 2012). En la misma estación de muestreo se capturó también $E$. serotinus, evidenciando la simpatría de ambas especies en esta zona. Por ello, parte de los registros sonoros asignados a $E$. serotinus en las cuadrículas más meridionales podrían corresponder a $E$. isabellinus.

\section{Nyctalus leisleri (Kuhl, 1817)}

Capturas en nueve cuadrículas, registros sonoros en otras diez. En total se aporta información para 19 cuadrículas, 14 de ellas nuevas (Fig. 19). Especie bien distribuida en la periferia de la comunidad, aunque solo puntualmente resultó abundante, como en el valle del río Arlanzón. Al tratarse de una especie en las que las hembras migran grandes distancias para llegar a sus áreas de reproducción al norte de Europa, el periodo del año en el que se realice el muestreo de esta especie condiciona en gran medida los resultados (Ibáñez et al. 2009). Durante el verano, únicamente los machos están presentes, mientras que a partir del mes de septiembre se observa un notable incremento de la población por la llegada de las hembras.

\section{Nyctalus lasiopterus (Schreber, 1780)}

Capturada en seis cuadrículas, todas nuevas. Se obtuvieron registros acústicos que podrían asignarse a esta especie en 13 cuadrículas (Fig. 20); no obstante, la diferenciación entre los registros acústicos de $N$. lasiopterus y N. noctula resulta dificultosa (Barataud 2012), por lo que no se han incluido en esta publicación.

Los datos disponibles en la bibliografía muestran que esta especie es común en el Sistema Central y en el Sistema Ibérico (Fernández 2002, Palomo et al. 2007) Durante la realización de este estudio resultó especialmente abundante en el Sistema Ibérico, tanto en la provincia de Burgos como en la de Soria.

\section{Barbastella barbastellus (Schreber, 1774)}

Se ha capturado en nueve cuadrículas y se han obtenido registros sonoros en otras siete. En total se aportan 14 nuevas cuadrículas, siempre en la periferia de la comunidad (Fig. 21). Los resultados muestran que es una especie común allí donde existen bosques de cierta entidad y mosaicos agropecuarios tradicionales bien conservados.

En 2011 se radiomarcaron dos hembras grávidas en el valle de Ancares (León) que utilizaron cuatro árboles diferentes en cinco días de seguimiento: tres Quercus pyrenaica y un Castanea sativa. En todos los casos utilizaron espacios bajo corteza, como es habitual en la especie (Simon et al. 2004, Steinhauser 2002, Drosera 2010), y ambas permanecieron en el mismo grupo. Un conteo realizado durante la emergencia del cuarto día de seguimiento arrojó un total de ocho ejemplares. En la localidad de Truchas (León) se observó también un ejemplar solitario refugiado durante el día bajo la corteza de un Quercus pyrenaica muerto.

\section{Plecotus auritus Linnaeus, 1758}

Se ha capturado en 13 cuadrículas, diez de ellas nuevas. Se han obtenido registros sonoros de Plecotus en cuatro cuadrículas, aunque no es posible diferenciar $P$. auritus/ austriacus con seguridad, por lo que no se han incluido dichas citas (Fig. 22). Los resultados muestran que es una especie común en los rebordes montañosos de la comunidad, como confirman también los datos bibliográficos (Fernández 2002, Palomo et al. 2007), pero escasea en las zonas más llanas. $P$. auritus es una especie que se refugia habitualmente en árboles (bajo cortezas o en árboles huecos por ejemplo) (Simon et al. 2004) si bien las agrupaciones de cría muestran una elevada dependencia de construcciones humanas en ciertas zonas de Europa (Swift 1998). En este estudio se han identificado dos refugios de cría en árboles: uno en un hueco en un Quercus robur en la ladera sur del valle de Ancares (León), a 1.249 m.s.n.m. y tan solo 1,5 m sobre el suelo; el otro en un agujero de pícido en un Castanea sativa en la localidad de San Vicente de la Cabeza (Zamora). Otro ejemplar, radiomarcado en el mes de septiembre en el valle de Ancares, utilizó un Quercus pyrenaica para refugiarse.

\section{Plecotus austriacus (Fischer, 1829)}

Se ha capturado en 12 cuadrículas, de las cuales nueve son nuevas (Fig. 23). Se identificó un refugio de cría de esta especie en una cabaña de pastores en el municipio de Hontoria del Pinar (Burgos). Especie común y bien distribuida en el ámbito de estudio. P. austriacus utiliza masas forestales para alimentarse (Swift 1998, Puig et al. 2009) y líneas de vegetación para desplazarse entre ellas. Durante la actividad nocturna puede alejarse más de $5 \mathrm{~km}$ del refugio (Simon et al. 2004, Puig et al. 2009). Por ello, la existencia de líneas de vegetación que doten de conectividad al paisaje resulta de gran importancia para esta especie. Esta conectividad es especialmente pobre en los páramos cerealistas, en los que las líneas de vegetación a menudo se limitan al cauce del río.

\section{Miniopterus schreibersii (Kuhl, 1817)}

Se ha capturado en dos cuadrículas, ambas nuevas: una en la sierra de Ancares y otra en la sierra de Peña de Francia (Fig. 24). No se han podido identificar con seguridad registros acústicos de esta especie. El hecho de que en Fernández (2002) no se recoja ninguna cita de $P$. pygmaeus en el ámbito territorial de este estudio (siendo ésta una especie muy común, como se ha visto), nos hace pensar en la posibilidad que en dicho trabajo se hayan atribuido parte de los registros de $P$. pygmaeus a $M$. schreibersii, de similares características.

\section{Tadarida teniotis (Rafinesque, 1814)}

Se han obtenido registros acústicos en nueve cuadrículas, seis de ellas nuevas (Fig. 25). En otras 40 cuadrículas se escucharon de oído posibles emisiones acústicas de esta especie, aunque no se obtuvieron grabaciones que permitiesen distinguirlas con seguridad de especies del género Nyctalus, por lo que no se han incluido en estos resultados.

Es una especie común y bien distribuida por el ámbito de estudio. 


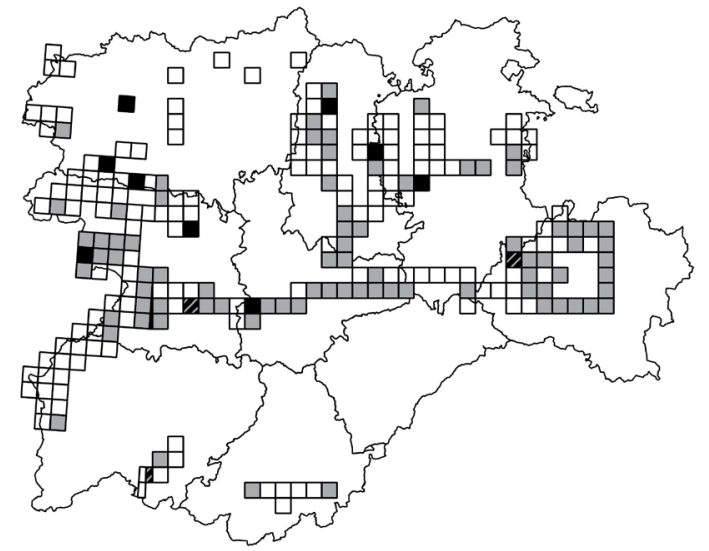

- Cuadrículas con presencia de la especie según bibliografía

- Nuevas cuadrículas aportadas en el presente trabajo

Cuadrículas en las que se había citado la especie en bibliografía

y se ha vuelto a detectar durante el presente estudio

Fig. 23 - Distribución de Plecotus austriacus en el área de estudio.

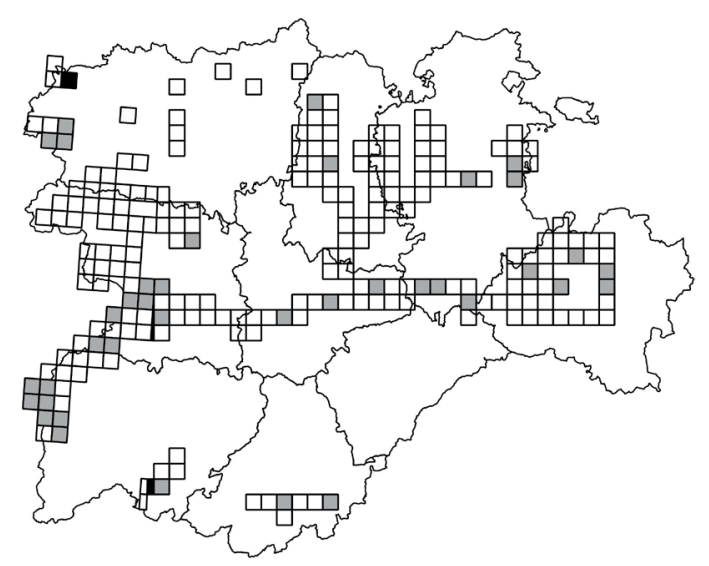

- Cuadrículas con presencia de la especie según bibliografía

- Nuevas cuadrículas aportadas en el presente trabajo

Cuadrículas en las que se había citado la especie en bibliografía y se ha vuelto a detectar durante el presente estudio

Fig. 24 - Distribución de Miniopterus schreibersii en el área de estudio.

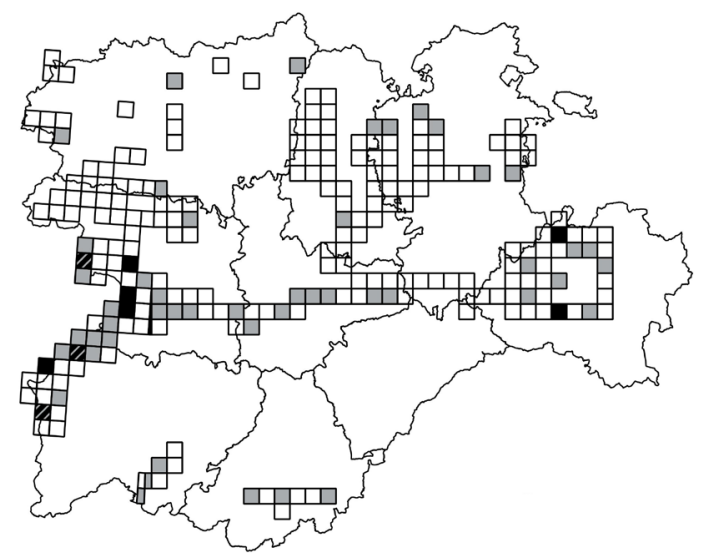

- Cuadrículas con presencia de la especie según bibliografía

- Nuevas cuadrículas aportadas en el presente trabajo

Cuadrículas en las que se había citado la especie en bibliografía $\checkmark$ se ha vuelto a detectar durante el presente estudio

Fig. 25 - Distribución de Tadarida teniotis en el área de estudio.

\section{CONCLUSIONES}

La diversidad de ambientes que alberga Castilla y León y la presencia de áreas montañosas bien conservadas, que suponen el encuentro de dominios climáticos diferentes, facilita que en esta comunidad existan comunidades diversas de murciélagos, siendo un buen ejemplo los resultados obtenidos en la montaña segoviana (de Paz et al. 2016, de Paz et al. 2017). Por su extensión, situación geográfica y diversidad de ambientes, Castilla y León es un territorio clave para la comprensión de la ecología y para la conservación de los murciélagos ibéricos.

De las zonas muestreadas en el presente trabajo, destacan por su diversidad y singularidad la sierra de Ancares y el norte de la provincia de Soria. Por lo que se refiere a los Ancares, solo en el valle del río Ancares se han detectado 13 especies de murciélagos, varias de ellas con evidencias de colonias reproductoras. En cuanto a la provincia de Soria, los pinares que se extienden al pie de los picos de Urbión albergan una nutrida población de N. lasiopterus, especie escasa y de gran interés en la península ibérica. En esta misma zona, la confluencia del pinar con manchas de bosque caducifolio favorece la presencia de especies poco abundantes en el ámbito de Castilla y León, como $M$. cf. nattereri o $B$. barbastellus. Y merece una especial mención el bosque aluvial y el mosaico silvopastoral situado entre Hinojosa de la Sierra y El Royo (Soria), en los que se ha comprobado la presencia de hembras reproductoras de tres especies forestales de gran interés: $M$. mystacinus, $M$. cf. nattereri y $B$. barbastellus. Sería de enorme interés conocer el estatus poblacional de estas especies en la zona, sus zonas refugio y áreas de alimentación.

El LIC Riberas del Tera y afluentes (Zamora) alberga una interesante fauna quiropterológica, con presencia de especies forestales como $M$. mystacinus, $M$. cf. nattereri, $N$. leisleri o B. barbastellus. En el LIC Río Aliste y afluentes (Zamora) la captura de hembras reproductoras de $B$. barbastellus y $P$. auritus, junto con la presencia de $M$. mystacinus, hace de esta zona protegida un espacio muy interesante para los murciélagos forestales. En el LIC Riberas del Río Alagón y afluentes (Salamanca) se ha constatado la presencia de $M$. mystacinus, $M$. bechsteinii y N. leisleri. Los bosques caducifolios que abundan en el valle del Alagón en los alrededores de la localidad de Mondragón deben ser tenidos en especial consideración. En el LIC Río Sil y afluentes (León), se pone de relieve la importancia de la existencia de una pequeña mancha de bosque aluvial en aceptable estado de conservación en la confluencia de los ríos Sil y Cúa-Burbia, en el que se han detectado hembras reproductoras de cuatro especies: $R$. ferrumequinum, $M$. daubentonii, $P$. pipistrellus y $P$. pygmaeus. Los bosques aluviales de las zonas bajas del río, más térmicas, constituyen un elemento clave para la reproducción de muchas especies de murciélagos cuando una extensión suficiente se mantiene en un adecuado estado de conservación. En el LIC Río Arlanzón y afluentes (Burgos) cabe destacar la presencia de $N$. leisleri, $N$. lasiopterus y $B$. barbastellus, tres especies forestales con áreas de distribución restringidas en el contexto ibérico. Y finalmente, en el LIC Riberas del Río Alberche y afluentes (Ávila) se citan tres especies forestales con áreas de distribución restringidas en el contexto ibérico 
(M. cf. nattereri, M. bechsteinii y N. lasiopterus) a las que hay que añadir $B$. barbastellus, citada en la bibliografía para este espacio.

En general, en la distribución de las especies de murciélagos en Castilla y León, se observa un patrón según el cual las especies más típicamente forestales aparecen fundamentalmente en el perímetro montañoso de la comunidad autónoma, pero están ausentes o son muy escasas en las zonas llanas desarboladas que ocupan buena parte de la zona central y de los exiguos bosques de ribera que atraviesan estas zonas. Esto puede ser debido a la pobreza del bosque de ribera en las zonas llanas, en las que con frecuencia queda reducido a una formación de galería a ambos lados del curso fluvial. Por otro lado, este exiguo bosque aparece habitualmente inmerso en una matriz de grandes extensiones de monocultivos que conforman un paisaje empobrecido, intensamente transformado y con poca conectividad. Las zonas montañosas, en las que el relieve complejo determina mayor diversidad de hábitats y mantiene cierta extensión y naturalidad de diferentes tipos de bosque, se han mostrado más diversas en cuanto a especies de murciélagos.

\section{AGRADECIMIENTOS}

Los muestreos realizados en 2011 se realizaron en el marco del proyecto "Murciélagos en soutos: un ejemplo de biodiversidad asociada a bosques culturales", financiada por la Fundación Biodiversidad y que contó con la colaboración de Novacaixagalicia.

Los muestreos realizados durante el año 2014 se enmarcaron en el "Estudio de la situación poblacional de los quirópteros dentro del ámbito del Proyecto LIFE11 NAT ES/699 MEDWETRIVERS (Riberas y humedales mediterráneos dentro de Red Natura 2000 en Castilla Y León)" coordinado por la SOMACYL

Las prospecciones realizadas en 2015 fueron financiadas por una Independent Research Fellowship del Natural Environment Research Council (NERC, UK), concedida a Orly Razgour (NE/M018660/1).

Para la captura de ejemplares se contó con los permisos pertinentes por parte de la Junta de Castilla y León.

En el trabajo de campo han colaborado Francisco Conde, Manuel Arzúa, Francisco Lamas, Paulo Barros, Sara Abalo, Sara Bachiller, Alba Remolar, Lara Teijeiro, Nerea Prieto y Luis Braz.

La identificación de especies crípticas mediante análisis de ADN ha sido posible gracias a Juan Luís García-Mudarra, Carlos Ibáñez y Javier Juste, del Laboratorio de Ecología Molecular (LEM) de la Estación Biológica de Doñana (CSIC).

Agradecemos a Javier Juste, Adrià López-Baucells y dos revisores anónimos las aportaciones realizadas para la mejora del presente artículo.

\section{REFERENCIAS}

AGUIRRE-MENDI, P. T, GARCÍA-MUDARRA, J.L, JUSTE, J. \& IBÁÑEZ, C. (2004). Presence of Myotis alcathoe Helversen \& Heller, 2001 (Chiroptera: Vespertilionidae) in the Iberian Peninsula. Acta Chiropterologica 6(1):49-57. http://doi. org/10.3161/1733-5329(2007)9[361:BOTRDM]2.0.CO;2

ALTRINGHAM J.D., BULLOCK D.J., WARREN R.D. \& WATERS D.A. (1997). The Biology and Conservation of Daubenton's bat, Myotis daubentonii. United Kingdom: Report for the Environment Agency.

BARATAUD, M. (2012). Écologie acoustique des chiroptères d'Europe. Biotope Éditions, Publications scientiques du Muséum national d'Histoire naturelle, Paris, France, 344 pp.

BARROS, P. (2012). Contribución al conocimiento de la distribución de quirópteros en el norte y centro de Portugal. Barbastella, 5(1): 19-31. http://dx.doi. org/10.14709/BarbJ.5.1.2012.04

CORONADO, A., FLAQUER, C., PUIG-MONTSERRAT, X., BARTGE, E., MAS, M., ARRIZABALAGA, A \& LÓPEZBAUCELLS, A. (2017). The role of secondary trees in mediterranean mature forests for the conservation of the forest-dwelling bat Myotis alcathoe. Are current logging guidelines appropriate? Hystrix, 28(2): 240-246. https://doi.org/10.4404/hystrix-00004-2017

DE PAZ, O., GONZÁleZ-ÁlVAREZ, F., \& MORENO, M.J. (2016). Los jardines del Palacio de La Granja (Segovia) ¿el lugar con mayor biodiversidad de quirópteros en la Península Ibérica? VI Jornadas SECEMU, 3-4 diciembre, 2016, Vairao, Portugal. https://doi.org/10.13140/ RG.2.2.13540.17285

DE PAZ, O., DE LA PEÑA, R., REDONDO, M., TENA, E. (2017). Bats of Valsaín Forest (Segovia, Spain): high species richness and activity use. 14th European Bat Research Symposium, 1-5 agosto, 2017, Donostia, España. https:// doi.org/10.13140/RG.2.2.19749.40165

DROSERA (2010). Murciélagos del Parque Natural Fragas do Eume. Inventario, distribución, ecología. Informe no publicado, $303 \mathrm{pp}$.

DROSERA (2014). Myotis mystacinus. En: Drosera. Morcegos de Galicia. Descargado el 01/05/2018.

FERNÁNDEZ, J. (2002). Los murciélagos de Castilla y León. Atlas de distribución y tamaño de las poblaciones. ed.: Junta de Castilla y León, España, 341 pp.

FITZSIMONS, P., HILL, D. \& GREENAWAY, F. (2002). Patterns of habitat use by female Bechstein's bats (Myotis bechsteinii) from a maternity colony in a British woodland. School of Biological Sciences, University of Sussex, Sussex, United Kingdom, p.1-21. 
FLAQUER, C., RUÍZ-JARILLO, R. \& ARRIZABALAGA, A. (2004). Contribución al conocimiento de la distribución de la fauna quiropterológica de Cataluña. Galemys, 16(2): 3955.

FLAQUER, C., PUIG-MONTSERRAT, X., BURGAS, A. \& RUSSO, D. (2008). Habitat selection by Geoffroy's bats (Myotis emarginatus) in a rural Mediterranean landscape: implications for conservation. Acta Chiropterologica, 10(1):61-67. https://doi. org/10.3161/150811008X331090

GALÁN, C. (1997). Fauna de Quirópteros del País Vasco. MUNIBE Ciencias Naturales, 49(1): 77-100.

GOITI, U., AIHARTZA, J., GUIU, M., SALSAMENDI, E., ALMENAR, D., NAPAL, M. \& GARIN, I. (2011). Geoffroy's bat, Myotis emarginatus, preys preferentially on spiders in multistratified dense habitats: a study of foraging bats in the Mediterranean. Folia Zoologica, 60(1): 17-24. https://doi.org/10.25225/fozo.v60.i1.a3.2011

HAARSMA, A-J. (2008). Manual for assessment of reproductive status, age and health in European vespertilionid bats. Publicación electrónica, 62 pp.

HERMIDA, R.J. \& ARZÚA, M. (2012). Utilización de refugios antrópicos por agrupaciones estivales de Myotis escalerai Cabrera 1904 en Galicia. IV Jornadas de SECEMU, Granollers, 8-9 de diciembre. https://doi.org/10.13140/ RG.2.2.31262.95044

HERMIDA, R.J., LAMAS, F.J., GRAÑA, D.A., RIAL, S., CERQUEIRA, F., ARZÚA, M., \& SEAGE, R. (2012) Contribución al conocimiento de la distribución de los Murciélagos (O. Chiroptera) en Galicia. Galemys, 24(1): 13-23. https://doi.org/10.7325/Galemys.2012.A02

HERMIDA, R.J., ARZÚA, M., SANTOS, L. \& LAMAS, F.J. (2013). Primeros datos sobre Myotis alcathoe en Castilla y León y primer refugio de cría en el noroeste de la península Iberica. Barbastella, 6(1): 30-33. http://dx.doi. org/10.14709/BarbJ.6.1.2013.04

HERNÁNDEZ, L. (2010). Murciélagos de la provincia de Salamanca. Conservación y estudio de los Murciélagos Salmantinos. Publicación online, 68 pp.

HUTSON, A.M., F. SPITZENBERGER, S. AULAGNIE \& COROIU, I. (2008). Myotis mystacinus. En: IUCN 2009. IUCN Red List of Threatened Species. Version 2009.2. Downloaded on 04 January 2010.

IBÁÑEZ, C., GARCÍA-MUDARRAJ.L., RUEDI, M., STADELMANN, B. \& JUSTE, J. (2006). The Iberian contribution to cryptic diversity in European bats. Acta Chiropterologica, 8(2): 277-297. https://doi.org/10.3161/17335329(2006)8[277:TICTCD]2.0.CO;2

IBÁÑEZ, C., GUILLÉN, A., AGIRRE-MENDI, P.T., JUSTE, J., SCHREUR, G., CORDERO, A.I. \& POPA-LISSEANU, A.G. (2009). Sexual segregation in Iberian noctule bats. Journal of Mammalogy, 90(1): 235-243. https://doi. org/10.1644/08-MAMM-A-037.1
JUSTE, J. (2017). Phylogeography of Daubenton's Bat Myotis daubentonii in Europe. 14th European Bat Research Symposium - EBRS 2017, 1-5 de agosto 2017, Donostia, España.

KAWAI, K., NIKAIDO, M., HARADA, M., MATSUMURA, S., LIN, LK., WU, Y., HASEGAWA, M. \& OKADA, N. (2002). Intraand interfamily relationships of vespertilionidae inferred by various molecular markers including SINE insertion data. Journal of Molecular Evolution, 55: 284-301. https://doi.org/10.1007/s00239-002-2326-0

LEUZINGER, Y. \& BROSSARD, C. (1994). Repartition de M. daubentonii en fonction du sexes et de la periode de l'annee dans le Jura Bernois. Mitt. Natf. Ges. Schaffhausen, 39: 135.

LISÓN, F., ALEDO, E. \& CALVO J.F. (2011). Los murciélagos (Mammalia: Chiroptera) de la Región de Murcia (SE España): distribución y estado de conservación. Anales de Biología, 33: 79-92.

LÓPEZ, Z. \& HERMIDA, R.J. (2016). Selección del hábitat de alimentación por Myotis bechsteinii (Kuhl, 1817) en los montes de O Morrazo, Pontevedra. Micolucus, 3: 103111.

MOLLEDA, R. \& FOMBELLIDA, I. (2018). Contribución al conocimiento de la distribución y estatus de la fauna quiropterológica de la Comunidad Autónoma de Cantabria. Journal of Bat Research \& Conservation, 11(1):XX-XX. https://doi.org/10.14709/ BarbJ.11.1.2018.04

NIERMANN, I., BIEDERMANN, M., BOGDANOWICZ, W., BRINKMANN, R., LE BRIS, Y., CIECHANOWSKI, M., DIETZ, C., DIETZ, I., ESTÓK, P., VON HELVERSEN, O., LE HOUÉDEC, A., PAKSUZ, S., PETROV, B.P., ÖZKAN, B., PIKSA, K., RACHWALD, A., ROUE, S.Y., SACHANOWICZ, K., SCHORCHT, W., TEREBA, A. \& MAYER, F. (2007). Biogeography of the recently described Myotis alcathoe von Helversen and Heller, (2001). Acta Chiropterologica, 9(2): $\quad 361-378 . \quad$ https://doi.org/10.3161/17335329(2007)9[361:BOTRDM]2.0.CO;2

NOGUERAS, J., GARRIDO-GARCÍA, J.A., FIJO-LEÓN, A., JUSTE, J., GARCÍA-MUDARRA, J.L. \& IBÁÑEZ, C. (2013). Patrones de distribución del complejo "Myotis mystacinus" en la Península Ibérica. Barbastella, 6(1): 23-29. http://dx.doi. org/10.14709/BarbJ.6.1.2013.03

PALOMO, L. J., GISBERT, J. \& BLANCO, J. C. (2007). Atlas y Libro Rojo de los Mamíferos Terrestres de España. Dirección General para la Biodiversidad-SECEM-SECEMU, Madrid, $588 \mathrm{pp}$.

PUIG, X., C. FLAQUER, R.G. RÀFOLS \& LÓPEZ, A. (2009). Selección de hábitat del orejudo gris (Plecotus austriacus) en ambientes litorales mediterráneos: importancia de los pinares y efectos del fuego sobre las poblaciones. IX Jornadas de la SECEM, 4-7 de diciembre, Bilbao, España. 
QUETGLAS, J. (2016). Murciélago ratonero ibérico - Myotis escalerai. En: Enciclopedia Virtual de los Vertebrados Españoles. Salvador, A., Barja, I. (Eds.). Museo Nacional de Ciencias Naturales, Madrid, España.

RUSSO D. (2002). Elevation affects the distribution of the two sexes in Daubenton's bats Myotis daubentonii (Chiroptera: Vespertilionidae) from Italy. Mammalia, 66(4): 543-551. https://doi.org/10.1515/mamm.2002.66.4.543

SALICINI, I., IBÁÑEZ, C., JUSTE, J. (2011). Multilocus phylogeny and species delimitation within the Nattere's bat species complex in the Western Palearctic. Molecular Phylogenetics and Evolution, 61(3): 888 -898. https:// doi.org/10.1016/j.ympev.2011.08.010

SALICINI, I., IBAÑEZ,C. \& JUSTE, J. (2013). El complejo Myotis nattereri en Iberia: una larga historia. Barbastella, 5(1): 3-7. http://dx.doi.org/10.14709/BarbJ.5.1.2012.01

SALSAMENDI, E., NAPAL, M., AIHARZTA, J., GOITI, U., ALMENAR, D. \& GARIN, I. (2007). Estudios de selección de hábitats en Myotis bechsteinii, Myotis emarginatus, Rhinolophus mehelyi y Rhinolophus euryale. Informe Final, Proyecto Life-Naturaleza "Conservación de quirópteros amenazados en Extremadura 2005-2008". SECEMU 2005/2007. Consejería de agricultura y medio ambiente. Junta de Extremadura, España.

SIMÕES, B.F., REBELO, H., LOPES, R.J., ALVES, P.C. \& HARRIS, D.J. (2007). Patters of genetic diversity within and beetween Myotis $d$. daubentonii and $\mathrm{M}$. $\mathrm{d}$. nathalinae derive from cytochrome b mtDNA sequence data. Acta Chiropterologica, 9(2): 379-389. https://doi. org/10.3161/1733-5329(2007)9[379:POGDWA]2.0.CO;2
SIMON, M., HÜTTENBÜGEL, S., \& SMIT-VIERGUTZ, J. (2004). Ecology and conservation of bats in villages and towns. Bundesamt für Naturschutz, Bonn, Germany, 263 pp.

SMITH, M.F., \& PATTON, J.L. (1993). The diversification of South American murid rodents: evidence from mitochondrial DNA sequence data for akodontine tribe. Biological Journal of the Linnean Society, 50(3): 149-177. https://doi.org/10.1111/j.1095-8312.1993.tb00924.x

STECK, C.E. \& BRINKMANN, R. (2006). The trophic niche of the Geoffroy's bat (Myotis emarginatus) in south-western Germany. Acta Chiropterologica, 8(2): $\quad 445-450$. https://doi.org/10.3161/17335329(2006)8[445:TTNOTG]2.0.CO;2

STEINHAUSER, D., BURGER, F., HOFFMEISTER, U., MATEZ, G., TEIGE, T., STEINHAUSER, P. \& WOLZ, I. (2002). Untersuchungen zur Ökologie der Mopsfledermaus, Barbastella barbastellus (Schreber, 1774), und der Bechsteinfledermaus, Myotis bechsteinii (Kuhl, 1817) im Süden des Landes Brandenburg. Schriftenr. Landschaftspflege Naturschutz, 71, 81-98.

SWIFT, S.M. (1998). Long eared bats. Publisher: T. \& A.D. Poyser, London, United Kingdom, 182 pp.

TUPINIER, Y. (1977). Description d'une chauve-souris nouvelle: Myotis nathalinae nov. sp. Mammalia, 41: 327 340. 